\title{
NIF System-Design Requirements for Nuclear-Weapons Physics Experiments
}

\author{
Theodore S. Perry \\ Lawrence Livermore National Laboratory \\ and \\ Bernhard H. Wilde \\ Los Alamos National Laboratory \\ Scientific Editors
}

\section{April 1995}
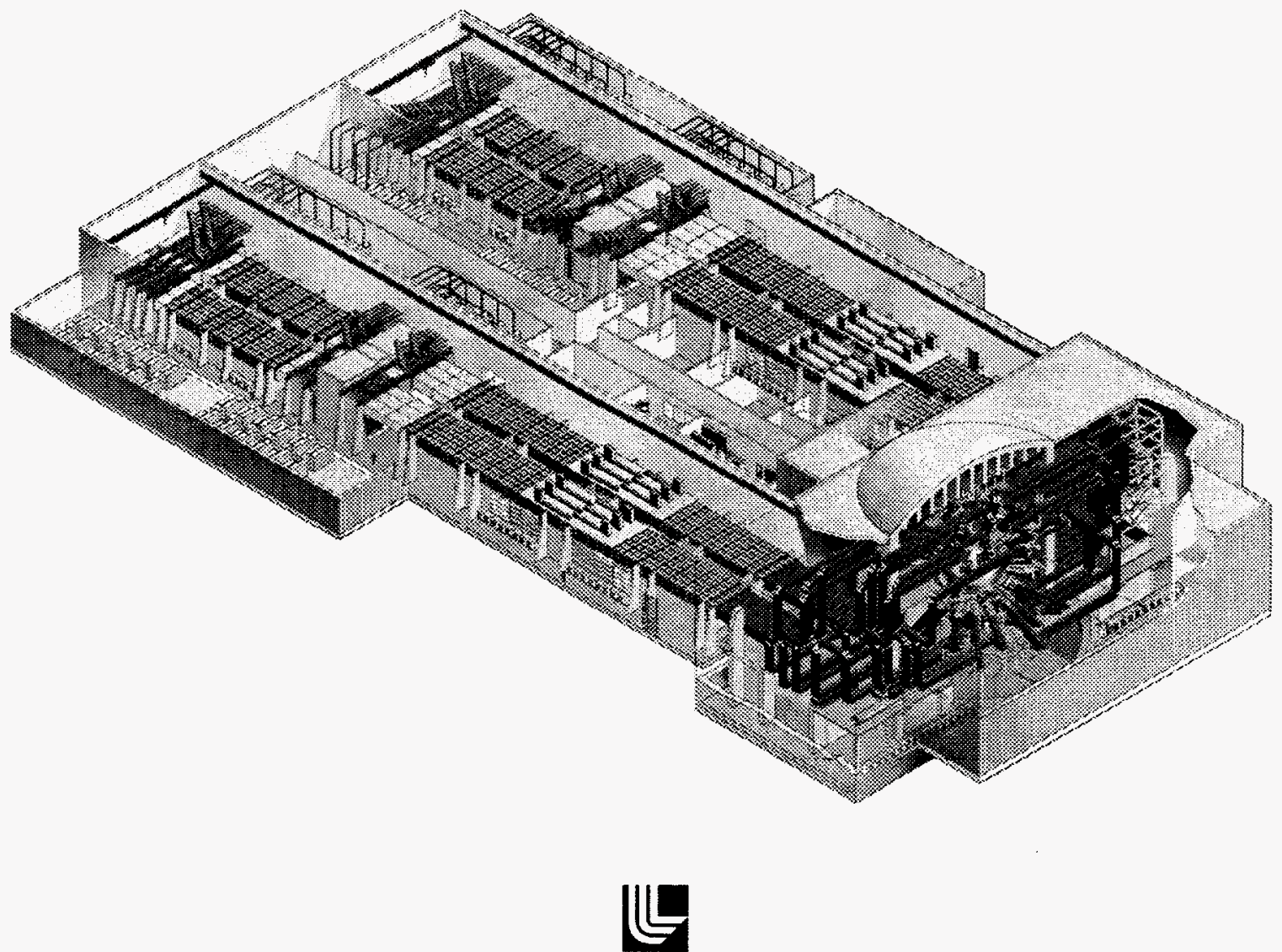

LAWRENCE LIVERMORE NATIONAL LABORATORY

University of California $\bullet$ Livermore, California $\bullet 94550$ 


\section{DISCLAIMER}

This document was prepared as an account of work sponsored by an agency of the United States Government. Neither the United States Government nor the University of California nor any of their employees, makes any warranty, express or implied, or assumes any legal liability or responsibility for the accuracy, completeness, or usefulness of any information, apparatus, product, or process disclosed, or represents that its use would not infringe privately owned rights. Reference herein to any specific commercial products, process, or service by trade name, trademark, manufacturer, or otherwise, does not necessarily constitute or imply its endorsement, recommendation, or favoring by the United States

Government or the University of California. The views and opinions of authors expressed herein do not necessarily state or reflect those of the United States Government or the University of California, and shall not be used for advertising or product endorsement purposes. 


\section{DISCLAIMER}

Portions of this document may be illegible in electronic image products. Images are produced from the best available original document. 


\title{
NIF System-Design Requirements for Nuclear-Weapons Physics Experiments
}

\author{
Theodore S. Perry \\ Lawrence Livermore National Laboratory \\ and \\ Bernhard H. Wilde \\ Los Alamos National Laboratory \\ Scientific Editors
}

April 1995

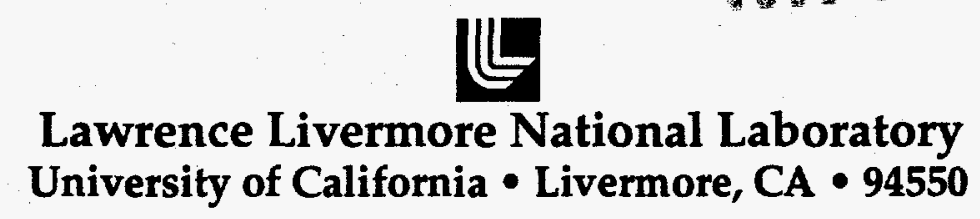

DISTRIBUTION OF THIS DOCUMENT IS UNLIMITED 


\section{NIF System-Design Requirements for Nuclear-Weapons Physics Experiments}

\section{April 1995}




\section{Table of Contents}

Foreword 7

I. Common Requirements for Weapons-Physics Experiments :................................................................. 9

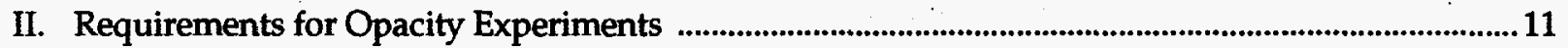

III. Requirements for Radiation-Flow Experiments ....................................................................................17

IV. Requirements for Equation-of-State Experiments ................................................................................. 19

V. Requirements for Non-LTE and X-Ray Laser Experiments ............................................................2 23

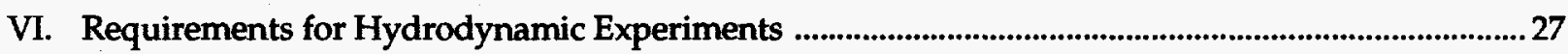

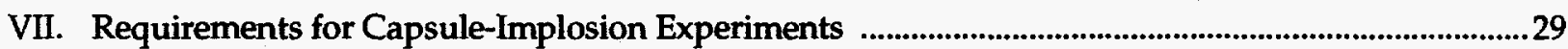




\section{Foreword}

\section{The role of NIF}

One of the primary objectives of the National Ignition Facility (NIF) project is to provide an aboveground experimental capability for conducting weapons-physics experiments, which is necessary for maintaining nuclear competence. The NIF is expected to produce the temperatures and pressures required for fusion ignition and to make significant contributions to the mission of DOE defense programs. To achieve the high-energydensity regimes needed for a science-based stockpile stewardship program, the NIF must be capable of producing conditions similar to those in nuclear weapon explosions. This qualifier imposes fundamental facility design requirements on the NIF. This document summarizes those requirements; full justification is included in the NIF white paper (Heidrich, 1995). This document concentrates on necessary additions to the current NIF Conceptual Design Report (CDR) (see UCRL-PROP-117093) if NIF is to fulfill its defense mission.

\section{Nuclear weapons physics and NIF}

The NIF will be an ideal facility for studying much of the physics involved in nuclear weapons both as isolated processes and as compound events. The NIF will be particularly important for studying opacity, radiation flow, equation of state, non-local thermodynamic equilibrium (non-LTE) physics, hydrodynamic instability, and fusion capsule design. Each of the weapons experiments designed for the NIF have their own requirements. The experimental plans and facility requirements unique to each area are reviewed in separate sections. Facility requirements from each of the experiments have been collected from the individual sections and listed in Section I, "Common Requirements for Weapons-Physics Experiments."

\section{Reference}

Heidrich, J. Ed., Weapons Physics on the NIF: Experimental Opportunities to Improve Predictive Modeling Capabilities of Nuclear Weapons Phenomena, Lawrence Livermore National Laboratory, Livermore, CA (UCRL-MI-119594) 1995.

The National Ignition Facility Conceptual Design Report, (CDR for the 192-beam facility) Lawrence Livermore National Laboratory, Livermore, CA (UCRL-PROP-117093) 1995. 


\section{Common Requirements for Weapons-Physics Experiments}

\section{Shot rate}

The weapons program anticipates approximately $\mathbf{1 8 0 0}$ shots in the first three to four years of NIF operation. This implies that NIF will have a sustained shot rate of six per day. Because not all the proposed shots will require the full energy of NIF, much useful work can be done while it is being brought up to full potential. For example, some experiments involving the development of new diagnostic techniques can start as soon as light is delivered to the target chamber. Thus, the weapons programs needs $\sim 50 \%$ of the available shots from the beginning of operations.

\section{Spot size}

All beams must be capable of focusing $50 \%$ of the laser energy into a spot with a 100 micron diameter and focusing $95 \%$ of the laser energy into a spot with a 200 -micron diameter for an average of 6 shots per day. In addition, it must be possible to switch in less than eight hours from the large smooth spots currently planned for ICF applications to the small spot sizes needed for weapon applications. Last, it must be possible to align each beam so that $40 \%$ of the energy hits a 100-micron fiber attached to a target inside the target chamber.

\section{Power}

To create the weapons conditions required for many experiments, the weapons program needs 1 megajoule (MJ) of 3w energy delivered in a 1-nanosecond (ns) pulse. Weapons program ignition-capsule experiments will require the standard $1.8 \mathrm{MJ}$ of energy, but the pulse will need to last for $5 \mathrm{~ns}$. A comprehensive laser design effort is necessary if both requirements are to be met.

\section{Target chambers}

The weapons program needs a second target chamber that is initially fed by 10 to $20 \%$ of the NIF beams. An additional design requirement will be the capability for upgrading this second chamber to provide it with all the capabilities of the first. This upgrade will permit sustaining a high shot-frequency rate by switching to the alternate chamber as the other cools.

\section{Laser wavelength}

The laser will deliver both $2 \omega$ and $3 \omega$ light to the center of each target chamber.

\section{Target positioners}

Three separate target positioners are needed; however, only one requires cryogenics.

\section{Pulse forming}

Pulse lengths will be variable from 100 picoseconds (ps) to 21 ns. Gaussian, square, rising, falling, and picket-fence-shaped pulses must be available. Each beam must be capable of independent timing (in groups of 4) and have up to 200-ns relative delays. 


\section{Line focus}

At least $20 \%$ of the beams must provide a $6-\mathrm{cm} \times 100-\mu \mathrm{m}$ line focus with $>10^{15} \mathrm{~W} / \mathrm{cm}^{2}$ at both the $2 \omega$ and $3 \omega$ energy levels.

\section{Stayout zone}

The ability to accommodate pinholes that are within $5 \mathrm{~cm}$ of the target is necessary.

\section{Unconverted light}

When operating at $3 \omega$, there must be a region extending perpendicularly away from the center of the hohlraum that is free from unconverted light. It must have at least a cylindrical shape that is $2 \mathrm{~mm}$ in diameter and 5 $\mathrm{cm}$ long. For other geometries, the ability to shield experimental packages external to the hohlraum from stray light must be maintained.

\section{Diagnostic stations}

There will be at least twelve, 12-in. manipulator tubes (TIM) with both complementary orthogonal views and views separated by about $20^{\circ}$ of latitude. These are required to increase the amount of data returned from each shot. The TIMs must be capable of supporting instruments weighing up to 500 pounds each. This will allow for $x$ - and gamma-ray shielding of instruments.

\section{Target alignment}

All target positioners must allow for target alignment with 5 degrees of freedom ( 2 rotations and 3 translations) within $\pm 10 \mu \mathrm{m}$ and $\pm 0.1^{\circ}$ of accuracy.

\section{Materials capabilities}

The facility must be capable of handling small amounts of uranium and other radioactive materials. The amounts required are small enough for NIF to maintain its designation as a non-nuclear facility. 


\section{Requirements for Opacity Experiments}

\section{Introduction}

The advent of a $>600$-terrawatt (TW) NIF will lead to the creation of laboratory conditions that nearly replicate some physical processes found in nuclear weapons. Based on simple scaling of the conditions measured at Nova with the NIF design calculations, it is clear that NIF will access qualitatively new physics and advance studies begun at Nova into regimes more directly relevant to secondary design. For example, radiative properties will be studied in opacity experiments using uranium ionized to the same electronic configurations found in a weapon, although at lower densities. For the first time in aboveground laboratories, hydrodynamic-mix experiments will last long enough to track instabilities from perturbative growth through turbulence at high compression. Also, for the first time, we can take equation-ofstate (EOS) measurements at pressures above a gigabar (Gbar), which will provide data in highenergy-density regimes. And finally, some integral experiments designed to simulate stockpile dynamics will use actual stockpile materials rather than lighter surrogates. If the NIF is equipped with a laser power that is less than $600 \mathrm{TW}$, it will significantly limit our ability to directly simulate conditions created by stockpile materials. Of course, scaled experiments using lower-density and lower- $Z$ materials will still have of some benefits.

\section{Role of opacity studies}

Opacities control the transport of radiative energy in nuclear weapons. As such, they affect many important aspects of weapons performance. Opacity models are complex, requiring knowledge of atomic structure, level populations, spectral-line shapes, and plasma interactions. Current models often rely on simplified assumptions and use approximations because of the enormous amount of atomic data required and the intractable nature of manybody problems. Opacity experiments are crucial for ensuring that our simplified models are reliable. Increased confidence in calculating opacities is essential to the goal of moving from an empirical to a predictive understanding of weapons performance.

\section{Nova opacity experiments}

To date, Nova experiments have provided highly resolved, frequency-dependent opacities for several mid-Z elements in LTE at temperatures from $10-60 \mathrm{eV}$ and densities around $1 \%$ of normal. In Nova experiments, an exceptional amount of effort went into simultaneously measuring the opacity, temperature, and density of plasmas. These experiments provided highly constraining benchmark data that significantly and unambiguously challenged the capabilities of our standard opacity codes. In response, new opacity models were developed for heavy elements. As a consequence, Nova experiments are driving new theoretical developments. The subsequent success of the new opacity models in explaining previous nuclear underground-test data represents a major demonstration of the role of laser experiments in improving the predictive capability of nuclear-design codes.

Because of the limited capabilities of Nova, opacity-modeling techniques have been benchmarked only in a fairly narrow temperature and density regime for a range of low-Z elements not directly applicable to weapons. In addition, significant uncertainties persist in the treatment of solid and higher densities, and of course, nothing can entirely take the place of measurements using actual stockpile materials, such as uranium. We have determined through straightforward extrapolation of techniques proven on Nova that 
NIF will be capable of extending our understanding of radiative opacities into these crucial regimes.

\section{NIF opacity experiments}

The advent of the NIF will significantly enhance the study of radiative opacities. Its ability to produce higher energy densities will permit accessing significantly higher temperatures and usher opacity research into qualitatively new regimes where radiation more nearly dominates the energy balance in a target. This provides opportunities to study relevant ions of high- $Z$ elements, including uranium, and plasma conditions approaching solid density. It also allows us to directly connect opacity data from laboratory experiments to opacity data from underground nuclear tests.

\section{Laser conditions}

Many areas of weapons-physics research, in addition to opacity studies, need hightemperature hohlraums to access material conditions relevant to weapons. Many also require higher powers, shorter pulses, and better focusing than those needed for ignition experiments. A study must be undertaken to identify a cost effective laser design that is consistent with achieving both ignition and high peak-focused power. Models indicate that for the hightemperature radiative environments produced by NIF, radiative losses through laser-entrance and diagnostic holes in hohlraums will begin to dominate hohlraum energetics. These losses can be minimized by making the targets and laser entrance holes small (provided this is consistent with maintaining laser-target coupling, obtaining suitable plasma conditions, and performing diagnosable experiments).

Opacity experiments on the NIF will be driven to equilibrium using radiative processes that mandate a hohlraum environment that minimizes radiative losses and produces a
Planckian radiation spectrum. In a model of a proposed NIF opacity experiment that used stockpile materials, we assumed the following: a laser power of $600 \mathrm{TW}$; a 1-ns square pulse; and a 200- $\mu \mathrm{m}$ spot size. Losses from laser back reflection, due to stimulated Brillouin scattering, were neglected. Our low-density uranium sample was stripped into an open-M-shell configuration and provided a target lifetime sufficient to establish both a steady state (i.e., nearly in LTE) and allow taking measurements. We found that even though laser-target coupling losses were not included and there were no provisions for backlighting, laser powers above 600 TW are probably necessary.

\section{Hohlraum conditions}

Approximately 50 NIF shots will be needed to develop, optimize, and characterize hightemperature hohlraum conditions. An additional 15 shots will be required to develop and optimize the high-intensity $x$-ray backlighters that produce an absorption spectra with hightemperature samples. Approximately 125 shots will be required for collecting actual opacity data. Most of the emphasis of these shots will focus on the emission spectra in the thermal region of the highest-temperature samples and include spectroscopic diagnostics of sample conditions and equilibrium. Some absorption experiments will explore the high-energy L-shell spectra of open-M-shell high-Z plasmas, where plasma emission does not complicate measurements. Opacity experiments in the thermal region will require simultaneously measuring both the emission and absorption spectra of moderate-temperature samples and then comparing these measurements to each other to ensure that LTE conditions are obtained. Finally, integral opacity measurements from foil burnthrough experiments will test energy balance and albedo questions related to the role of wall loss in radiation flow. Foil burnthrough measurements will also access conditions up to near solid density. 


\section{Proposed opacity experiments*}

\section{Creating and characterizing high- temperature hohlraums}

1.1. Energy coupling and plasma instabilities

- Laser requirements:

Energy $=500 \mathrm{~kJ}$.

Pulse $=1$ ns, square shaped (or equivalent) with fewer beams.

- Number of shots: 10.

- Special diagnostics:

Optical and $x$-ray spectrometers, $x$-ray imaging, and calorimetry.

- Goals:

Confirm models and perform scaled experiments on laser-matter energy coupling at the high-intensity short scale length needed to create hightemperature radiation environments. Measure intensity of laser back reflection from stimulated Brillouin scattering (SBS), stimulated Raman scattering (SRS), and test models for laser coupling and conversion efficiency to $x$ rays.

- Deliverables:

Characterize and test models for laser-matter coupling to guide the design of high-temperature hohlraums and provide understanding of hohlraum energetics.

1.2. Hohlraum filling and lifetime

- Laser requirements:

Energy $=500 \mathrm{~kJ}$.

Pulse $=1 \mathrm{~ns}$, square shaped.

- Number of shots: 20.

- Goals:

Test designs (vacuum or gas/foam fill) for limiting hohlraum closure, providing optimal laser coupling, and optimizing target lifetime.

- Deliverable:

Provide a proven hohlraum design with a lifetime that allows for target heating, equilibration, and taking measurements.

\footnotetext{
* Approximately 210 shots for the first three years.
}

\subsection{Hohlraum conditions}

- Laser requirements: Energy $=500 \mathrm{~kJ}$.

Pulse $=1$ ns, square shaped.

- Number of shots: 20.

- Goals:

Measure the x-ray-drive spectrum, time-dependent equivalent temperature, shock breakout, and average ionization in high- $Z$ foam targets. Optimize the high-temperature hohlraum design models used to produce a nearly Planckian spectrum and LTE conditions.

- Deliverable: Provide the highest temperature hohlraum to date with a characterized Planckian spectrum that allows for the smallest corrections to any nonequilibrium process.

\section{Backlighter techniques development ${ }^{*}$}

- Laser requirements: Energy $=200 \mathrm{~kJ}$. Pulse $=500$ ps, square shaped.

- Number of shots: 10.

- Goals:

Develop methods for providing broadband high-brightness backlighters needed for absorption measurements both in the multi-kilovolt range and in the thermal spectral regions of highradiation-temperature plasmas.

- Deliverables:

High-energy backlighters for measuring absorption in the multikilovolt regime where target emission is small. Highradiation-temperature backlighters for spectroscopically measuring the Rosseland mean opacity for the highest possible temperatures.

\footnotetext{
${ }^{\text {** }}$ Shots are for technique validation and may take place during the NIF phase-in period.
} 


\section{High-temperature emission experiments using optimized hohlraums}

- Laser requirements:

Energy $=700 \mathrm{~kJ}$.

Pulse $=1 \mathrm{~ns}$.

Risetime $=10-90 \%$ in $<100$ ps.

Spot size $=200 \mu \mathrm{m}$.

- Number of shots: $\mathbf{5 0}$

- Goal:

Measure the thermal emission spectra of well-characterized, high-temperature high- $Z$ targets. The plasma conditions and equilibrium will be determined by using spectroscopic diagnostics and tracer elements. Sample conditions and materials will vary.

- Deliverables:

High-resolution measurements of the LTE emission spectra and high-Zsample conditions. Data should allow reasonable estimates of the Rosseland mean opacities (which are needed for calculating radiative heat flow) and provide highly detailed spectroscopic measurements of opacity. These are also necessary for testing the accuracy of approximations used in opacity models. This should allow for nearly direct comparisons with existing underground nuclear-test data.

\section{High-temperature inner-shell absorption experiments}

- Laser requirements:

Energy $=700 \mathrm{~kJ}$.

Pulse $=1 \mathrm{~ns}$.

Risetime $=10-90 \%$ in $<100$ ps.

- Number of shots: 25 .

- Goals:

Measure the L-shell (inner-shell) absorption spectra of well characterized, open-M-shell high- $Z$ samples using high-energy $x$-ray backlighters in a spectral region where the sample emission is low. Test the inner-shell absorption spectra as a possible diagnostic of plasma conditions.
- Deliverables:

Inner-shell absorption spectra data that can be used for testing aspects of linebroadening models used for outer shells that strongly influence radiative heat transfer. Develop plasma diagnostic techniques for other measurements.

\section{High-temperature spectroscopic emission and absorption opacity measurements in the thermal region}

- Laser requirements:

Energy $=700 \mathrm{~kJ}$.

Pulse $=1 \mathrm{~ns}$.

Risetime $=10-90 \%$ in $<100$ ps.

Spot size $=200 \mu \mathrm{m}$.

- Number of shots: 50 .

- Goal:

Measure the thermal emission and absorption spectra of well characterized, moderate-temperature high- $Z$ targets.

High-radiation-temperature thermal backlighters will be required to measure the absorption of the highesttemperature samples. Proximity to LTE conditions will be verified by applying Kirchhoff's law, which relates the emission and absorption spectra. Plasma conditions (including radiation and electron temperatures, electron density, and the population distribution of atomic energy levels) will be determined using spectroscopic diagnostics and tracer elements. Sample conditions and materials will vary. The $x$-ray backlighting requirements in the thermal region will restrict measurements to lower temperature samples and necessitate scaling results to materials and conditions relevant to weapons.

- Deliverables:

High-resolution measurements of LTE emission and absorption spectra and conditions for high-Z samples. Data should allow for accurate determinations of Rosseland mean opacities, which are necessary for calculating radiative heat flow. Data should also provide highly detailed 
spectroscopic measurements of opacity, which are necessary for testing the accuracy of approximations used in opacity models. Using both emission and absorption spectra to characterize conditions will improve the accuracy of the data, ensure that the data meets quality standards, and provide the data needed to determine if these are appropriate for LTE opacities.

\section{High-Z burnthrough wedge foils}

- Laser requirements:

Energy $=700 \mathrm{~kJ}$.

Pulse $=1 \mathrm{~ns}$.

Risetime $=10-90 \%$ in $<100$ ps.

Spot size $=200 \mu \mathrm{m}$.

- Number of shots: 25.

- Goal:

Measure the rate of radiation-energy penetration and the propagation of a Marshak wave in a high- $Z$ foil by comparing spectral brightness versus time as a function of sample thickness using a high- $Z$ wedge target. With careful drive characterization, this will be an integral measurement of the Rosseland mean opacity at high densities. This can also be used to identify energy-loss mechanisms, a requirement for understanding radiation flow.

- Deliverable:

Enhanced understanding of radiation penetration versus albedo in high- $Z$ materials, which is needed for testing energy-loss mechanisms and radiationflow models in weapons.

\section{For further information, contact}

William H. Goldstein (510) 422-2515, or Paul T. Springer (510) 423-9112. 


\section{Requirements for Radiation-Flow Experiments}

\section{Introduction}

Radiation-flow experiments on NIF will study the transport of energy by $x$ rays through one-, two-, and three-dimensional (1-D, 2-D, and $3-D)$ geometries. Energetic $x$-ray drives will be needed to create regimes where the relative contributions of radiation and material energy densities can be varied as much as possible. Considerable flexibility in laser irradiation conditions and diagnostic positioning will also be required to investigate complicated 2- and 3$D$ radiation-flow geometries.

\section{Special system and diagnostic requirements}

1. SOP (ultraviolet) and adjacent FXI/SXI (x-ray) diagnostic ports capable of supporting simultaneous, almost colinear, views of shock breakouts from the rear surfaces of foils.

2. A short, 1-ns, pulse delivering $1 \mathrm{MJ}$ of laser energy at $3 \omega$ to drive energetic hohlraums.

3. A gas-fill system that delivers $1-10$ atmospheres of gases such as krypton, xenon, or radon. The gas-fill reservoirs and delivery tubes must be thermostatically controllable at temperatures up to $\sim 70^{\circ} \mathrm{C}$ and capable of containing chemically corrosive gases such as $\mathrm{UF}_{6}$.

4. Shielding for experimental packages external to the hohlraum to protect them from stray laser light.

5. Complementary diagnostic views.

6. A Dante-like instrument with channels sensitive to higher photon energies.
7. A 200- $\mu \mathrm{m}$ spot size for smaller, hotter hohlraums and brighter $x$-ray backlighters.

8. The capability for placing diagnostic pinholes within a $5-\mathrm{cm}$ radius of the target.

\section{Proposed radiation-flow experiments*}

\section{Hohlraum characterization}

- Number of shots: 60.

- Goal:

Detailed characterization of the $x$-ray drive from a variety of hohlraum sources. This is of prime importance for design and diagnosis of NIF radiationflow experiments. Problems related to shot-to-shot reproducibility should be carefully examined.

- Deliverables:

Measurement of the time-, frequency-, and angular-dependence of the $x$-ray spectra produced by different NIF hohlraum drivers. Both cylindrical and spherical hohlraums will be used as well as possible alternative hohlraum designs. The production of hot, Planckian spectra will be optimized.

\section{One-dimensional burnthrough foils}

- Number of shots: 50 .

- Goal:

Simultaneously measure the shock breakout and $x$-ray radiative burnthrough from high- $Z$ foils. This data can be used to constrain theoretical high-Z opacity models.

\footnotetext{
* Estimates by topic (total number of shots, $~ 350$ ) for the first three years.
} 
- Deliverable:

Measurements of the time-, frequency-, and 1-D spatial dependence of radiation and shock-front propagation through foils with different atomic numbers. This data will be used in conjunction with opacity code calculations and radiation hydrodynamic simulations of shock- and Marshak-wave propagation.

\section{Two-dimensional radiation flow in} high-Z tubes

- Number of shots: 80 .

- Goal: Characterize radiation flow through cylindrical tubes.

- Deliverables: Measurements of the radiation flow down tubes as a function of: opacity of wall materials, tube length, and x-raydrive strength. A variety of materials of interest will be experimentally quantified for use in conjunction with code simulations.

\section{Radiation flow in more complicated geometries}

- Number of shots: 80 .

- Goal:

Characterize radiation flow through 2- and 3-D geometries.

- Deliverable:

Measurement of the time- and spatialdependence of radiation and material temperatures at various locations in complicated geometries.

\section{Mass ablation rates of $x$-ray-driven foils}

- Number of shots: 50 .

- Goal:

Study a suite of materials with different atomic numbers and sample densities to quantify the 1-D process describing the $x$-ray heating and subsequent radiation hydrodynamics caused by $x$-ray ablation by materials. This study will also include interaction at boundaries of layered materials.

- Deliverable: Measurement of the time- and spatialdependence of the density and temperature of $x$-ray-ablated materials (both doped and undoped) using a variety of initial material densities, compositions, and $\mathrm{x}$-ray drive conditions.

\section{Advanced diagnostic development}

- Number of shots: 30 .

- Goal:

Develop advanced diagnostic techniques for a variety of radiationflow experiments.

- Deliverable: New capabilities and experimental methods.

\section{For further information, contact}

Ted Perry (510) 423-2065, or

Richard Ward (510) 423-2679. 


\section{Requirements for Equation-of-State Experiments}

\section{Introduction}

An equation of state (EOS) is a mathematical expression of the fundamental relationship between pressure, density, and temperature of a material. An accurate EOS describes how materials react and interact and is crucial to any hydrodynamic description of a system.

All weapons performance and effects simulations and laser experiments supporting the stockpile stewardship program require accurate equation-of-state information in regimes where experimental data are very sparse or non-existent. For most materials, some fundamental (interpretation-independent) data may be available at pressures less than $5 \mathrm{Mbar}$. However, at higher pressures there are only sparse interpretation-dependent data available for a few materials. (Most of these are based on impedance-matching data.) Virtually all EOS data required for stockpile-stewardship applications are in high-pressure regimes that are far above $5 \mathrm{Mbar}$. Because calculations require EOS information over a large parameter space encompassing pressure, density, and temperature, EOS models providing interpolation and extrapolation must be used. We use experiments to gather baseline data that is used to validate models. Our experimental data, to date, show inconsistencies and errors in our EOS database.

High-energy-density EOS are needed for stockpile stewardship and laser applications in these particular areas:

- Implosion dynamics.

- Single- and multiple-shock timing.

- Radiation-matter interactions.

- Ionization and thermal-wave propagation.

- Hydrodynamics.

- Effects on diagnostics in high-energydensity environments.

\section{Program guidelines}

Equation-of-state experiments on the NIF will concentrate on the region of maximum uncertainty, which is typically in the 3- to 1000 Mbar range. Below $3 \mathrm{Mbar}$, accurate data is available from gas-gun experiments. At high pressures, the Thomas-Fermi theory is accurate, although 1000 -Mbar is probably still too low for Thomas-Fermi analysis of high- $Z$ metals.

Presently, fundamental data is only available under $5 \mathrm{Mbar}$. However, by the time NIF is on line, this regime may have been extended through the use of other exotic schemes or by Nova experiments. In any case, some data will have to be obtained in NIF low-pressure regimes to normalize it to known data and thus begin a bootstrap method of collecting EOS data for higher-energy-density regimes.

We will begin by obtaining fundamental data for standard materials (i.e., materials used in impedance-matching experiments). Experimental data for standard materials must be as code- or model-independent as possible with regard to interpretation.

Measuring temperatures in EOS experiments has a high priority because temperature and density are explicit variables in both EOS and opacity models. We have learned that:

- Temperature is a more sensitive test of EOS models.

- There is higher uncertainty surrounding temperature than pressure or density.

- Temperature is a more sensitive measure of shock stability than velocity.

Determining the temperature of shocked. and compressed matter is a problem yet to be solved, and it is expected to remain difficult. However, experiments in this area can provide an enormous amount of EOS information. 
We expect that to conduct EOS measurements at $1 \mathrm{Gbar}$, we will need shocks stronger than $10 \mathrm{Gbar}$. With the planned NIF capabilities, this can only be done using smallscale hohlraums, beams at nearly full energy levels, and a laser spot size smaller than $200 \mu \mathrm{m}$.

Further metrology for EOS targets will require routine measurements of $0.1 \%$ flatness over wedges and step plates that are tens-ofmicrons thick.

\section{Proposed EOS experiments}

The anticipated program has been divided into seven areas. Additional notation regarding required instrumentation is included at the end of this section.

\section{Method characterization}

- Number of shots: 20.

- Goal:

Evaluate methods of steady shock production and shock uniformity in direct, indirect, and hohlraum drives and measure the $x$-ray preheat of targets. (If a temperature diagnostic is developed on Nova, before NIF is operational, it will be evaluated on NIF during these shots.)

- Special requirements: $X$-ray laser (XRL) interferometer or a Nova-demonstrated reflectometry setup, which is as yet untested.

- Deliverables:

1. Quantified demonstration of uniform single-shock breakout using three different drives each with timing benchmarked to code predictions. These experimental results will lead to the selection of a preferred method for driving different types of experiments. 2. Measurement of preheat due to ambient $x$ rays from heating source in targets.

3. Measurement of preheat ahead of a shock created by radiation emitted from the shock front.

\footnotetext{
* Approximately 405 shots over 5 years.
}

\section{Temperature diagnostic development}

- Number of shots: 20.

- Goal:

Evaluate emission and absorption spectroscopy used to infer electron temperatures between 10 and $100 \mathrm{eV}$ in compressed (> solid density) matter.

- Special requirements: A sub-picosecond time-resolved streak camera operating in the ultraviolet spectrum with a hard x-ray (i.e., 4- $7 \mathrm{keV}$ ) continuum backlighter.

- Deliverables:

1. A spectroscopic method for evaluating temperatures of shocked matter.

2. Identification of a preferred method for driving EOS experiments.

\section{Hugoniot measurements of fundamental materials}

- Number of shots: 100.

- Goal:

Obtain interpretation-independent Hugoniot curves for standard materials.

- Special requirements: A temperature diagnostic and subpicosecond time-resolved streak camera.

- Deliverables:

Principal Hugoniot data in the 1-Mbar to 1-Gbar range for aluminum, molybdenum, and gold.

\section{Hugoniot measurements of secondary materials}

- Number of shots: $\mathbf{3 0}$ shots per material (200 shots minimum).

- Goal:

Obtain interpretation-independent (impedance-matched) Hugoniot curves for additional materials.

- _. Special requirements: A temperature diagnostic and a subpicosecond time-resolved streak camera.

- Deliverables:

Principal Hugoniot data in the 1-Mbar to 1 - Gbar range for plastic $(\mathrm{CH})$, solid deuterium-tritium, uranium, and plutonium. 


\section{Release experiments}

- Number of shots: 40 .

- Goal:

Investigate off-Hugoniot data of released isentropes.

- Special requirements:

XRL interferometer setup and also temperature diagnostics.

- Deliverables:

Released isentrope data for deuteriumtritium and gold.

\section{Isochoric heating experiments}

- Number of shots: 15 (5-10 require ignition).

- Goal:

Investigate off-Hugoniot data of nonablative isochoric heating by neutrons and $K \alpha$ line photons.

- Special requirements: Ignition hohlraums, extremely highintensity laser source, XRL interferometer, and temperature and neutron diagnostics.

- Deliverable:

Evaluation of short-burst $\mathrm{K} \alpha$ heating, neutron heating of thin samples and comparisons of EOS models.

\section{Exotic experiments}

- Number of shots: 10 .

- Goal:

Generate extremely high pressures using plasma jets or colliding flyer plates to approach the regime where the Thomas-Fermi theory is valid.

- Special requirements: Extremely high intensity backlighter, XRL interferometer setup, and temperature diagnostic.

- Deliverable: Comparison to Thomas-Fermi theory of inferred pressures in the $>10$ Gbar regime.

\section{Required instrumentation}

- Goal:

Develop a high-speed high-dynamicrange streaked camera.

- Equipment specifications: Time resolution $=200$ fs for very high velocity shocks. For many experiments, $100 \mathrm{fs}$ resolution is preferred. Dynamic range $=>50$.

This camera needs to operate in both the UV and XUV spectrum, (i.e., $4-150 \mathrm{eV}$ ), but not simultaneously.

- Special requirements: A pulsed optical-laser interferometry/ reflectometry station on the equator that is either movable or fixed at $90^{\circ}$. The optical laser must be capable of producing at least $100 \mathrm{~mW}$ with pulse lengths greater than $10 \mathrm{~ns}$.

\section{Additional considerations}

It will be necessary to have an instrumentation diagnostic checkout facility so that full-system shots are not wasted on this required, but mundane, task. It seems that there are three options:

1. Perform checkouts only as ride-alongs to other experiments.

This is the least favorable option, but the ability to perform instrument debugs as ride-alongs must be available.

2. Perform checkouts at another, but comparable facility.

This may mean using the Omega facility in Rochester, NY, which would be approximately adequate for our needs, or using the second target chamber at the megajoule laser facility in France.

3. Build a second target chamber at LLNL similar to the French design.

The third option is the most flexible, but presumably the most expensive. However, a second target chamber at LLNL would provide the opportunity for conducting lower-intensity (Nova energy level) experiments in parallel with higher-energy shots. 
For the EOS program, a second target chamber would be used for technique development and diagnostic checks. However, it is anticipated that most EOS shots involving data collection will be done with high drive energies. The NIF drive energy is at least an order of magnitude smaller than required to reach the Thomas-Fermi regime in high- $Z$ materials. This means that the approach to Thomas-Fermi (high-energy-density) measurements needs to be done with the highest drive energy available. Accuracies in measurements at lower-energy density will be limited by experimental configuration conditions. Improving the accuracy of measurements requires larger targets (spatially larger shocks over longer times), therefore, even for these measurements, the high drive energy will be used.

Note that, most of the technique and diagnostic development experiments listed can take place during the NIF phase-in period.

\section{For further information, contact}

Robert Cauble (510) 422-4724. 


\section{Requirements for Non-LTE and X-Ray Laser Experiments}

\section{Introduction}

The NIF will allow us to study high-energydensity physics that cannot be accessed in the absence of underground nuclear testing. The following is a proposed three-year experimental plan for non-LTE $x$-ray laser experiments on NIF, totaling $\mathbf{2 0 0}$ shots. These are needed to maintain our core competency in non-LTE design and to develop shortwavelength $x$-ray lasers for dual-benefit applications. To perform $x$-ray laser research on NIF, we must have narrow $(\sim 100 \mu \mathrm{m}$ at FWHM) line-focused beams available.

Our plan includes developing a testbed for non-LTE physics, exploring new regimes and schemes in $x$-ray laser research, and using $x$-ray lasers for plasma-imaging diagnostics. These lasers are used for plasma imaging because they provide absolute plasma-density measurements. This information is needed to validate codes and benchmark data for both the ICF and weapon-physics communities. In this report we describe the goals, plans, and facility requirements for each type of experiment over a three-year period.

\section{Proposed plasma imaging experiments}

- Pulse requirements:

Shaped and delivered on target at least $200 \mathrm{~ns}$ before the main beams arrive.

- Number of shots: 50 .

- Special diagnostics: X-ray laser

- Stay-out zone for $x$-ray laser target: Depends on ability to shield the primary target from the backlighter beams and debris.

- Alignment for $x$-ray laser imaging: Use an in-situ technique.

- Facility requirements:

Single-sided line-focused illumination that is $6 \mathrm{~cm} \times 100 \mu \mathrm{m}$ and $>10^{15} \mathrm{~W} / \mathrm{cm}^{2}$ at $2 \omega$ (until we demonstrate $x$-ray laser operation at $3 \omega)$.

- Goals:

With its short wavelength (35-400 $\AA$ ), controllable short-pulse duration, highpeak brightness, and spatial and temporal coherence, the $x$-ray laser is ideally suited as a plasma diagnostic for imaging rapidly evolving ( $<1 \mathrm{~ns}$ ) laserdriven plasmas with high electron densities $\left(10^{21} \mathrm{~cm}^{-3}<\mathrm{n}_{\mathrm{e}}<10^{24} \mathrm{~cm}^{-3}\right)$. We have recently applied technological advances in multilayer mirrors and beam splitters to the soft $x$-ray regime and used the unique properties of $x$-ray lasers to develop a soft-x-ray laser interferometer that operates at $155 \AA$ on Nova. The continued development and application of short-wavelength $x$-ray lasers to probe rapidly evolving highdensity laser plasmas on NIF is of great interest to both the ICF and weaponphysics communities. The shots in this series of experiments are needed to test $x$-ray laser configurations for interferometry applications using different types of targets in a variety of experimental setups.

- Deliverable:

The ultimate objective is to develop a reliable diagnostic instrument for the ICF and weapon-physics communities. A short-wavelength $x$-ray laser interferometer will permit measuring 2-D electron-density profiles. This 
information is important for benchmarking and validating code physics in numerical simulations.

\section{Proposed ionization balance experiments}

- Number of shots: $\mathbf{5 0}$.

We propose using 20 shots to characterize the $x$-ray spectrum of the source and the backlighter. The remaining 30 shots study the temporal evolution of uniform plasmas, such as argon and krypton gas cells.

- Facility requirements: These point-projection backlighter experiments use a hohlraum as the radiation source and include a backlighter beam. The backlighter beam needs to be focused to a spot of the order of $100 \mu \mathrm{m}$ in diameter with intensities on the order of $10^{16} \mathrm{~W} / \mathrm{cm}^{2}$.

- Goal:

Develop a radiatively driven gas or foam cell, which will be used to develop a testbed for photoionization and recombination kinetics and radiation transfer in non-LTE plasmas. Using either a hohlraum or other $x$-ray converters, such as a high- $Z$ slab, we can irradiate and photoionize spatially uniform plasmas. We can then measure ionization states of these plasmas using inner-shell point-projection absorption spectroscopy. Potentially, this testbed could be used to measure radiationtransfer properties of optically thick plasmas and line-profile variations of optically thick lines using a cavity design. By carefully controlling experimental configurations, we can use this testbed to calibrate our codes.

- Deliverable:

Development of a testbed for non-LTE physics and the initial study of non-LTE physics issues relevant to high-energydensity regimes.

\section{New regimes and schemes for $x$-ray lasers}

- Pulse requirements: Picket-fence shape, including the prepulse, with traveling-wave capability.

- Number of shots: 100 .

- Facility requirements:

Double-sided line-focused illumination that is $6 \mathrm{~cm} \times 100 \mu \mathrm{m}$ and $>10^{16} \mathrm{~W} / \mathrm{cm}^{2}$ on each side at $2 \omega$ (until we demonstrate $x$-ray laser operation at $3 \omega$ ). A set of the backlighter beams capable of pointing $\pm 15 \mathrm{~cm}$ away from the primary target to test long $x$-ray laser architectures. These long lasers deliver double-sided line-focused illumination that is $6 \mathrm{~cm}$ $\times 100 \mu \mathrm{m}$ and $>10^{15} \mathrm{~W} / \mathrm{cm}^{2}$ on each side at $2 \omega$. Delays should be of the order of $1 \mathrm{~ns}$.

- Goal: Gain understanding of the physics of short wavelength $x$-ray lasers and $x$-raylaser architectures required to produce more efficient and brighter $x$-ray sources. The large energy available on NIF will allow us to extend existing $x$-ray-laser schemes into new regimes and to test new schemes for producing short-wavelength $x$-ray lasers. The addition of flexible pointing, in the $x-y$ direction, will allow us to study extreme saturation effects and to test $x$-ray-laser architectures, such as the oscillatoramplifier configurations.

- Deliverable:

Development of an efficient, bright, and coherent $x$-ray source is important in the study of non-LTE plasmas. These shots are needed to test new schemes and to explore new x-raylasers regimes. Ultimately, the information gathered will be used to study various laser architectures and test architectural components.

* Similar to the requirements for radiation-flow experiments in Section III. 


\section{Other facility requirements}

\section{Second target chamber}

An additional target chamber, with up to $25 \%$ of all the beams able to be directed at the target chamber, would allow us to test diagnostics with greater flexibility and faster shot turn around.

\section{Contributing authors}

Alan S. Wan, Peter Celliers, Robert Cauble, Luiz B. Da Silva, Jeffrey A. Koch, Stephen B. Libby, Richard A. London, Juan C. Moreno, Joseph Nilsen, and Rosemary S. Walling.

\section{For further information, contact}

Stephen B. Libby (510) 422-9785, Joseph Nilsen (510) 422-4766, or Alan S. Wan (510) 423-3342. 


\section{Requirements for Hydrodynamic Experiments}

\section{Introduction}

Experiments for the NIF hydrodynamic program will look at instability behaviors in two and three dimensions. They will also be used to study instabilities, develop advanced experimental techniques, and examine other topics of hydrodynamic interest. Requirements for hydrodynamics experiments include the following:

1. A long drive pulse, lasting more than $25 \mathrm{~ns}$.

2. Flexibly timed, independently pointable beams that can be used to make $x$-ray backlighting sources. The pointable beams must contain up to $500 \mathrm{~kJ}$ of laser energy and must be capable of being delayed up to $40 \mathrm{~ns}$.

3. Pinholes that can be placed within a 5$\mathrm{cm}$ radius of the target, as needed.

4. Shielding from stray light, as needed, for experimental packages located external to the hohlraum.

5. Diagnostic stations that provide complementary orthogonal views of the target are very desirable. These will increase the amount of data returned by each shot.

6. Target alignment with 5 degrees of freedom ( 2 rotations and 3 translations) within $\pm 10 \mu \mathrm{m}$ and $\pm 0.1^{\circ}$ of accuracy.

7. A smaller laser-focus spot (i.e., 200- $\mu \mathrm{m}$ diameter). As many as $25 \%$ of the experiments could benefit from the capability to produce a smaller focused spot.

8. A second target chamber, with energy levels on the order of $200 \mathrm{~kJ}$. This could be used to test diagnostics and new configurations and to conduct related experiments that do not require the full power of the NIF. This last request, while expensive, is necessary if we are to properly utilize the NIF for these types of experiments.

\section{Proposed hydrodynamic experiments*}

\section{Rough-surface Richtmyer-Meshkov instability}

- Number of shots: 60 .

- Goal: Investigate Richtmyer-Meshkov instability, at internal interfaces, in a regime that has small, rough initial perturbations driven through large amplification.

- Deliverable: Measurement of the development of the mixing region as a function of time for varying Atwood numbers, shock strengths, and surface roughnesses.

\section{Rough-surface Rayleigh-Taylor instability}

- Number of shots: 40 .

- Goal: Investigate Rayleigh-Taylor instability, at internal interfaces, in a regime that has small, rough initial perturbations driven through large amplification.

- ... Deliverable: Measurement of the development of the mixing region as a function of time for varying Atwood numbers, acceleration speeds, and surface roughnesses.

\footnotetext{
${ }^{*}$ A total of $\sim 490$ shots over three years.
} 


\section{Convergent 2-D instabilities}

- Number of shots: 40.

- Goal:

Study instabilities and mixing in 2-D convergent geometries.

- Deliverable:

Measurement of instability growth in a 2-D convergent geometry for different drive conditions, initial perturbations, and cylindrical geometries.

\section{Three-dimensional (3-D) instabilities}

- Number of shots: $50^{*}$.

- Goal:

Study instabilities and mixing in 3-D geometries.

- Deliverable:

Measurement of instability growth in a 3-D geometry for different drive conditions, initial perturbations, and geometric configurations.

\section{Atomic-scale turbulent mixing}

- Number of shots: $50^{*}$.

- Goal:

Study small-scale mixing in both turbulent and non-turbulent regimes.

- Deliverable: Measurement of the fine-scale (atomic) mixing and analysis of its dependence on a variety of parameters including: surface roughnesses, Atwood numbers, and acceleration/shock conditions.

\section{Large-scale flows}

- Number of shots: 75.

- Goal: Investigate large-scale structures, such as jets and shear layers, found in a variety of flows in an environment characterized by high temperature, high density, and high Mach number.

- Deliverable: Experimental quantification of a variety of flows of interest for use in conjunction with code simulations.

\footnotetext{
* Some will use ignition.
}

\section{Advanced topics}

- Number of shots: $75^{*}$.

- Goal:

Develop advanced diagnostic techniques for use on a variety of hydrodynamics issues, such as radiochemistry, deuterium-tritium reactions, and direct drive.

- Deliverable:

New capabilities that can address other hydrodynamics issues, such as the measurement of atomic mixing.

\section{Developmental work}

- Number of shots: $100^{* *}$.

- Goals:

Develop new diagnostics, methods, and configurations for NIF experiments, as well as some sub-NIF experiments (with the addition of a second chamber).

- Deliverables:

New diagnostic capabilities and experimental methods that have been tested under realistic conditions and demonstration of proof-of-principle for high-risk ideas. Additionally, a second chamber would provide a matched facility for pursuing parameter variations over a much larger range than is logical for NIF. For example, quantities such as low-end shock strength could be explored in a range that doesn't require the full energy of NIF, but would, at the same time, benefit from the NIF diagnostics, laser characteristics, and facility peculiarities, etc. Not everything will have been done in the earlier Nova regime leaving a portion of parameter space between NIF and Nova for experimentation.

\section{For further information, contact}

Guy Dimonte (510) 423-0596,

Paul Miller (510) 423-6455, or

Tom Peyser (510) 423-6454.

\footnotetext{
** Most could be conducted in the second target chamber.
} 


\section{Requirements for Capsule-Implosion Experiments}

\section{Overview of shot plans*}

The facility design requirements for weapons-physics capsule experiments are similar to those for ICF capsules (see the NIF Conceptual Design Report). The description, justification, and deliverables for these experiments are necessarily contained elsewhere; consequently, this section just summarizes the experimental shot plans.

- Ignition capsules containing uranium, 40 shots.

- X-ray output from ignition capsules, 40 shots.

- Sub-ignition capsules containing uranium, 40 shots.

- Special effects, 140 shots.

Note that because 160 of these are full-yield shots, they will only be possible after ignition and gain are demonstrated. They therefore will need to be scheduled three to four years after the first ignition experiments. Additional diagnostics required for these experiments will also include neutron spectrometers with spectral coverage from $100 \mathrm{keV}$ to $20 \mathrm{MeV}$, electromagnetic-pulse detectors, and radiochemistry procedures for recovering most of the initial mass in the capsule for analysis.

\section{For further information, contact}

Ted Perry (510) 423-2065 or

Dana Rowley (510) 422-9675.

\footnotetext{
* Approximately 260 shots.
} 